\title{
A New Method to Recover Vectorial Electric Fields and Current Densities from Unstructured Meshes
}

\author{
DANIEL C. KERR ${ }^{a^{*}}$ and ISAAK D. MAYERGOYZ ${ }^{\mathrm{b}}$ \\ ${ }^{a}$ Department of Electrical Engineering University of Maryland, College Park, MD 20742, ${ }^{b}$ Department of Electrical Engineering University \\ of Maryland, College Park, MD 20742
}

\begin{abstract}
In the context of 2-D and 3-D unstructured mixed-element meshes, a new method of recovering vectorial fields and currents in multidimensional simulation is introduced. The new method, called the method of edge elements, directly interpolates the projections of the vectors on the edges of an element into its interior. The new method is compared to two other recovery methods on the basis of resolution, consistency, and implementation ease.
\end{abstract}

\section{INTRODUCTION}

In the numerical simulation of semiconductor devices, the vectorial electric field and current density are required throughout the domain of simulation. During the simulation, they are needed to compute various physical parameter models, such as mobility and impact ionization. When the simulation has finished, they are again needed for post-processing and visualization. The problem is to recover the vectorial fields and currents from the nodal values of electric potential, quasi-Fermi potentials, and temperatures. The problem is complicated by the unstructured mesh and the special discretizations of current. In finite-box simulations, the discretization and solution do not uniquely define the fields off of the edges joining the nodes. Recovery methods must be developed to reconstruct the vectorial electric field and current density from their projections along edges of the unstructured mesh.
For accuracy, the ideal recovery method should be of high resolution and consistent with the model and its discretization. Resolution measures the capability to distinguish between fields at adjacent locations. Higher resolution enables higher-order discretizations ofphysical models, which can reduce the truncation error. Inconsistency will introduce additional numerical errors. The recovery method should be consistent with the approximations of the Scharfetter-Gummel (SG) discretization. The recovered field should be in agreement with the data input to the method, so that the projections of the recovered field on the element edges reproduce the original data.

For computer efficiency, the ideal recovery method should be easy to implement and applicable to any 2-D or 3-D, unstructured, and mixed-element mesh. A mixed-element mesh is a mesh which contains simplices as well as non-simplices, i.e., in 2-D, rectangles as well as triangles, and in 3-D, cuboids, prisms, pyramids, and tetrahedrons. The implementation of the

* Present address. National Semiconductor Research Laboratory, 2900 Semiconductor Drive, Mail Stop E-100, Santa Clara, CA 955052-8090, USA. Tel: (408) 721-1700 Email: dankerr@ampere.nsc.com 


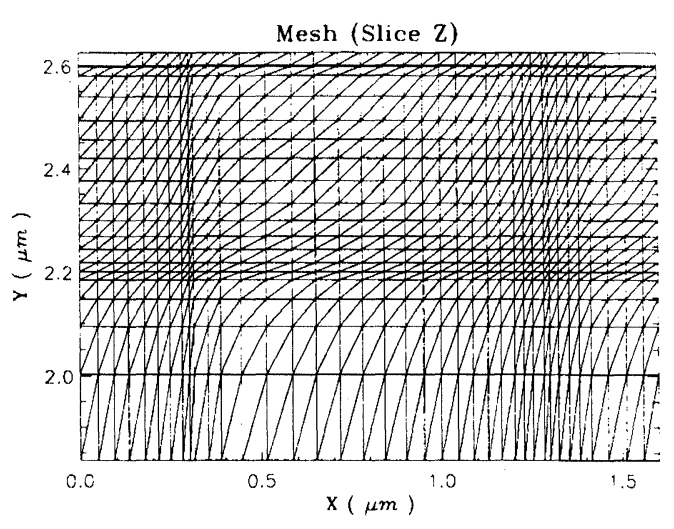

(a) One of the split meshes.

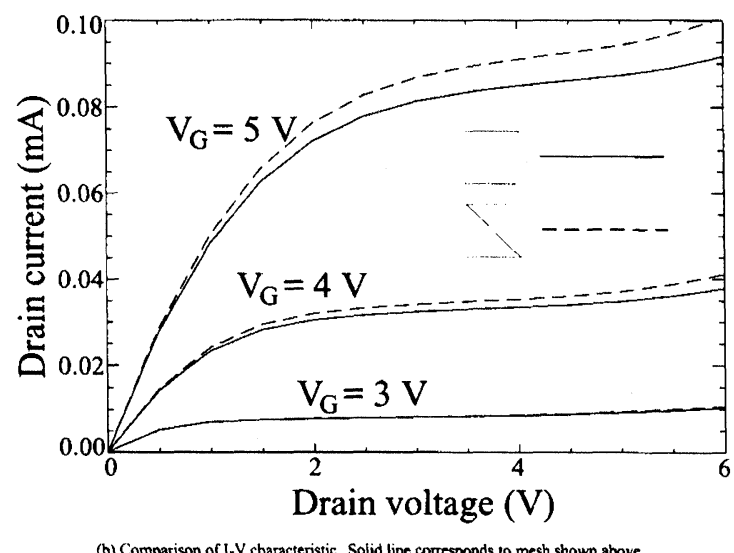

FIGURE $1 \mathrm{I}-\mathrm{V}$ curves calculated for a 2-D $1.2 \mu \mathrm{m}$ MOSFET. The simulation was performed using a tensor-product mesh in which each rectangle was split into two right triangles in two different ways, as indicated. The simulation used simple field-dependent mobility model and impact ionization

method should be computationally inexpensive, including the calculation of derivatives of the field for the Jacobian.

Often, in order to apply a method only suitable for simplex meshes, nonsimplex elements are arbitrarily subdivided into simplices. However, the calculations, illustrated in Fig. 1, show the problem with this. Namely, it turns out that the numerical solutions are sensitive to the way elements are split. Splitting the elements introduces new edges $i j$ with zero Voronoi cell areas $A_{i j}$, which in no way influences the structure of the discretized equations. Errors introduced in calculating the fields will feed back into the solution via the physical models, which may compromise the overall accuracy of the modeling effort. Although the magnitude of this problem will diminish as the mesh is refined, more accurate recovery methods can extract the needed information without arbitrary splitting. Errors introduced in calculating the fields will feed back into the solution via the physical models, which may compromise the overall accuracy of the modeling effort.

Here, the new field-recovery method is described, which is called the modified edge-element method [1]. Then, two other methods, the methods, of least-squares fitting and method of corner averages, are reviewed and the methods are compared.

\subsection{Method of Edge Elements}

The method of edge elements (EEM) directly interpolates vectorial values defined on the edges of an element into the interiorof the element. The vectorial interpolant $\mathbf{J}^{\mathrm{k}}$ of the edge values $F_{i j}$ into the interior of element $\mathrm{K}$ is

$$
\mathbf{J}^{k}=\sum_{i j \in \varepsilon^{k}} F_{i j} \mathbf{e}_{i j}
$$

where $\mathbf{e}_{i j}$ is the basis function of edge $i j$ and $\varepsilon^{k}$ is the set of edges of the element. The edge basis function is defined by

$$
\boldsymbol{e}_{i j}=d_{i j}\left(\lambda_{i} \nabla \lambda_{j}-\lambda_{j} \nabla \lambda_{i}\right)
$$

where $d_{i j}$ is the length of edge $i j$ and $\lambda_{i}$ is the scalar finite-element shape function. The shape function $\lambda_{i}$ for the element takes the value 1 at node $i$, the value 0 at all other nodes, and is linear between them. The basis function has the following characteristic properties: the tangential component of $\boldsymbol{e}_{i j}$ along the edge $i j$ is equal to one, while the tangential components along all other edges are equal to zero. Reconstruction of the field using edge elements yields a non-constant vector function defined on the element which has the following properties: (1) The projection of the edge -element reconstruction on each edge of the element reproduces the original data. (2) The tangential components of the field are continuous from one element to the next. (3) The field is divergence-free. Property (2) is useful at internal interfaces between materials 
TABLE I The two-dimensional edge elements in standard position, each normalized by its length. A modified edge-element is shown in the vector plots, with node numbering indicated

\begin{tabular}{ccccc|c}
\hline \hline & \multicolumn{2}{c}{ Standard EE } & \multicolumn{2}{c}{ Modified EE } & Element averaged \\
Element & $\hat{\mathbf{a}}_{x}$ & $\hat{\mathbf{a}}_{y}$ & $\hat{\mathbf{a}}_{x}$ & $\hat{\mathbf{a}}_{y}$ & \\
\hline Triangle & & & & & $<\mathbf{J}_{x}^{k}>=\frac{1}{3}\left(2 d_{01} F_{01}+d_{02} F_{02}-d_{12} F_{12}\right)$ \\
$\mathbf{e}_{01}$ & $1-y$ & $x$ & $1-y$ & $x$ & \\
$\mathbf{e}_{02}$ & $y$ & $1-x$ & $y$ & $1-x$ & $<\mathbf{J}_{y}^{k}>=\frac{1}{3}\left(d_{01} F_{01}+2 d_{02} F_{02}+d_{12} F_{12}\right)$ \\
$\mathbf{e}_{12}$ & $-y$ & $x$ & $-y$ & $x$ & \\
Rectangle & & & & & $<\mathbf{J}_{x}^{k}>=\frac{1}{2}\left(d_{01} F_{01}+d_{23} F_{23}\right)$ \\
$\mathbf{e}_{01}$ & $(1-y)^{2}$ & 0 & $1-y$ & 0 & \\
$\mathbf{e}_{02}$ & 0 & $(1-x)^{2}$ & 0 & $1-x$ & $<\mathbf{J}_{y}^{k}>=\frac{1}{2}\left(d_{01} F_{01}+d_{23} F_{23}\right)$ \\
$\mathbf{e}_{13}$ & 0 & $x^{2}$ & 0 & $x$ & \\
$\mathbf{e}_{23}$ & $y^{2}$ & 0 & $y$ & 0 & \\
\hline
\end{tabular}

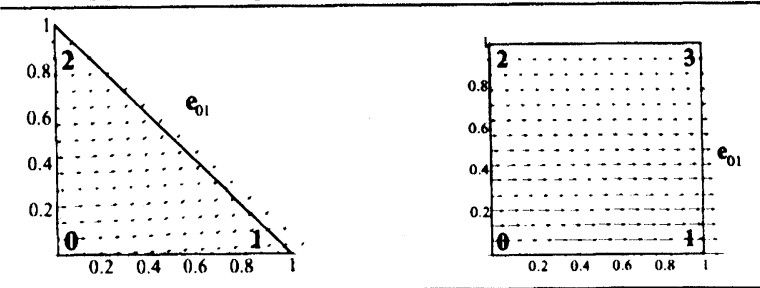

of different permittivities. Property (3) is consistent with the approximations made for the SG discretization of current.

To simplify the edge-element calculations, a coordinate transformation is applied to transform each element into its standard position. The coordinate transformation for an element with nodes at $\left\{\mathrm{r}_{0}, \mathrm{r}_{1}, \ldots\right\}$ into standard position is defined by $\mathrm{r}^{\prime}=A^{-1}\left(\mathrm{r}-\mathrm{r}_{0}\right)$, where $A$ is the Jacobian of the transformation. The edge elements transform according to

$$
\mathbf{e}_{i j}(\mathbf{r})=\left(A^{-1}\right)^{T} \mathbf{e}_{i j}\left(\mathbf{r}^{\prime}\right),
$$

where the primed coordinates refer to the element in standard position.

Although the definition of the edge elements can be applied to non-simplex elements, the results are inaccurate due to the quadratic component in the direction normal to the edge. To rectify this, the modified edge-elements are introduced by removing one of the factors from each squared term. In this form, edge elements are suitable for 2-D and 3-D nonsimplex elements. The standard and modified 2-D edge-elements in standard position are listed in Table I.
The vector quantity at a location required for a parameter model may be computed by averaging the interpolant function. For example, analytically averaging over the element $k$ results in

$$
<\mathbf{J}^{k}>=\frac{1}{V^{k}} \int_{V^{k}} \mathbf{J}^{k} d v
$$

where $V^{k}$ is the volume of the element $k$. On the other hand, the space-varying interpolant function may be directly evaluated at the location of interest.

\section{METHOD OF LEAST6SQUARES FITTING}

The method of least-squares (LSM) fitting treats the edge values in an element as measurements of the field. The recovered field is a constant field within the element that minimizes the error along each edge, that is, the field $\mathbf{J}^{k}$ that minimizes $f\left(\mathbf{J}^{k}\right)$,

$$
f\left(\mathbf{J}^{k}\right)=\sum_{i j \in \varepsilon^{k}}\left(\mathbf{J}^{k} \cdot \hat{\mathbf{a}}_{i j}-F_{i j}\right)^{2}
$$


where $\hat{\mathbf{a}}_{i j}$ is a unit vector from node $i$ to node $j$. The LSM can be applied uniformly to edge-, element-, or cell-field recoveries.

\section{METHOD OF CORNER AVERAGES}

The method of corner averages (CAM) partitions the domain into "corners" and calculates a constant vector value in each corner. The volume $V_{i}^{k}$ associated with the corner of element $k$ at node $i$, where node $i$ belongs to the element, is defined by the intersection of element $k$ with the Voronoi cell at $i$. The corner value $\mathbf{J}_{i}^{k}$ in $V_{i}^{k}$ is calculated by solving the system of equations

$$
\mathbf{J}_{i}^{k} \cdot \hat{\mathbf{a}}_{i j}=F_{i j}, \quad j \in N_{i}^{k},
$$

where $\mathcal{N}_{i}^{k}$ is the set of nodal neighbors of $i$ within element $k$.

The field at a location required for a parameter model is computed through a suitably weighted average of corner values. The original CAM [1] was proposed for triangular or prismatic elements, and it is difficult to generalize their averaging scheme to other elements. Here, a more general averaging scheme is proposed which is suitable for other elements. The generalized average is defined by

$$
<\mathbf{J}>=\sum_{i, k} \mathbf{J}_{i}^{k} / V_{i}^{k} / \sum_{i, k} 1 / V_{i}^{k},
$$

where the indices $(i, k)$ take on different values depending on whether the average is for a node, an element, or along an edge.

\section{DISCUSSION}

The EEM was compared to the LSM and the CAM. On the basis of resolution, the EEM is clearly superior since it can distinguish any two arbitrarily close points. The other methods produce fields which are constant over various zones. On the basis of consistency, the EEM is consistent with the SG discretization. The recovered field is also consistent with the solution, since the projections ofthe field along element edges reproduces the original data. However, the other methods do not have this property. On the basis of implementation ease, the EEM and LSM are computationally cheaper that the CAM. The work involved in recovering the field using the EEM or LSM involves evaluating a polynomial function in the number of edges and multiplying by a matrix for the coordinate transformation.

A detailed comparison between the element-averaged EEM and the LSM was made, since it can be argued that the LSM produces fields which are optimum. It was found that the two methods yield identical results for the rectangular faces of any element, so the differences were evaluated in triangular faces. For an equilateral triangle, with nodes at $(0,0),(1,0)$, and $(1 / 2, \sqrt{ } 3 / 2)$, the recovered fields are identical and given by

$$
\begin{aligned}
\mathbf{J}^{k}=\left\{0.667 F_{01}+0.333 F_{02}\right. \\
\left.-0.333 F_{12}, 0.577 F_{02}+0.577 F_{12}\right\} .
\end{aligned}
$$

When the upper node was moved upward, from $(1 / 2$, $\sqrt{ } 3 / 2)$ to $(1 / 2,10+\sqrt{ } 3 / 2)$, the methods produced :

$$
\begin{array}{r}
\mathbf{J}_{E E M}^{k}=\left\{0.667 F_{01}+3.62 F_{02}-3.62 F_{12},\right. \\
\left.0.501 F_{02}+0.501 F_{12}\right\}, \\
\mathbf{J}_{L S M}^{k}=\left\{0.996 F_{01}+0.045 F_{02}-0.045 F_{12},\right. \\
\left.0.501 F_{02}+0.501 F_{12}\right\},
\end{array}
$$

These methods differ in the reconstruction of the $x$-component of the field. Which is correct? The EEM uses the geometry of the figure when averaging over the area of the element. Longer edges dominate more of the figure and therefore weigh proportionately more in the averaging. On the other hand, the LSM ignores the geometry of the figure in which the field is computed; only the relative orientation of the measurements matters. In the elongated triangle, the upper legs are rotated toward the $y$-direction and thus the influence of these legs on $J_{x}^{k}$ is smaller. This means that the LSM is inaccurate for element reconstructions. The use of the LSM should be restricted to field reconstructions where all the measurements are collected at one point, for example, to reconstruct the field at the center of the Voronoi cell. 
In conclusion, the newly proposed method of edge-elements is an accurate and efficient vectorial field reconstruction method. The EEM has been installed in SIMASTER, a general purpose 2-D and 3-D device simulator using unstructed meshes [2]. The EEM has proven effective in many different types of simulations.

\section{References}

[1] S. E. Laux and R. G. Byrnes, "Semiconductor device simulation using generalized mobility models," IBM J. Res. Develop., vol. 29, no. 3, pp. 289-301, 1985.

[2] D. C. Kerr and I. D. Mayergoyz, "Simulation of semiconductor devices using the fixed-point iteration method on 3-D unstructured meshes," submitted to IEEE Trans. Computer-Aided Design, 1995. 

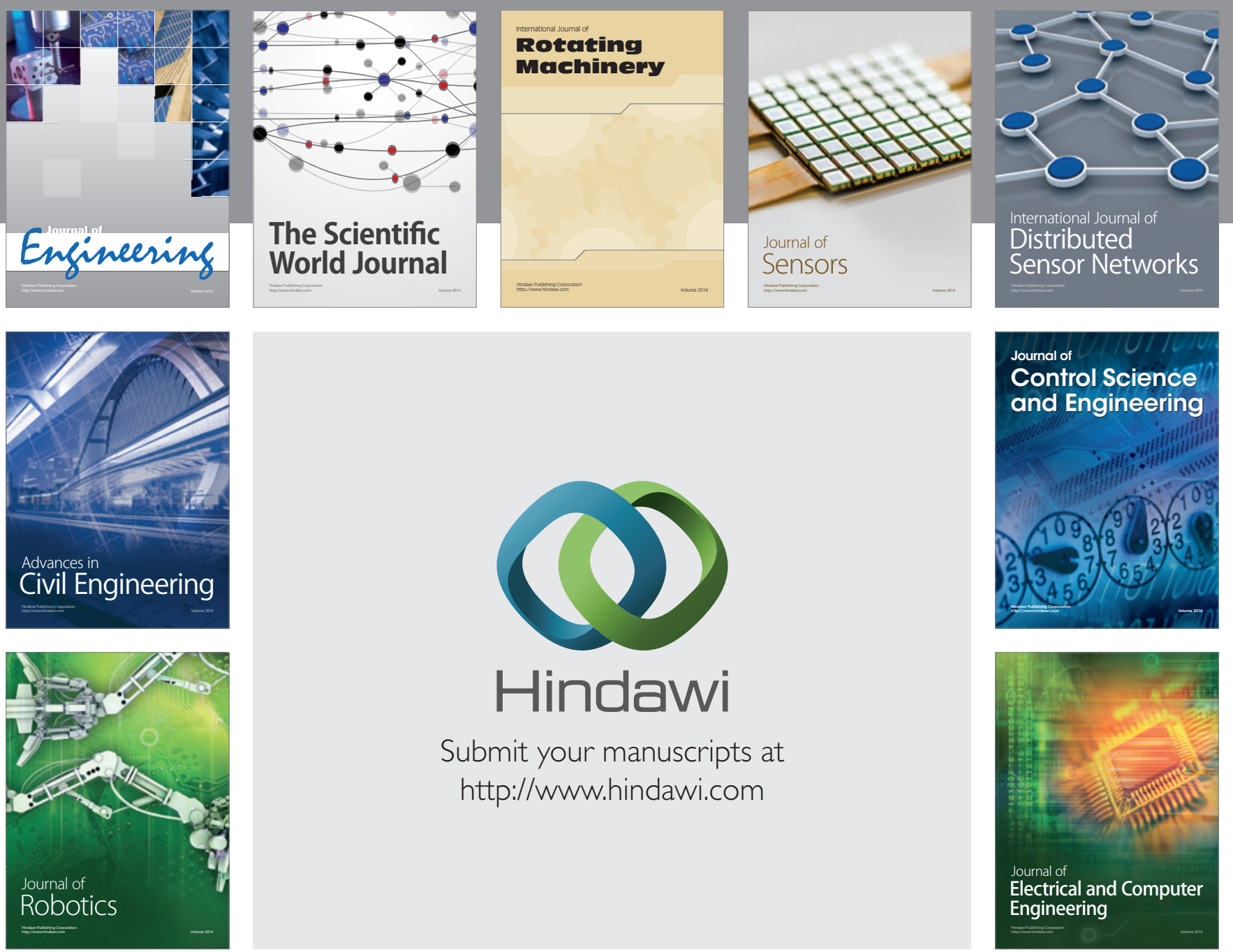

Submit your manuscripts at

http://www.hindawi.com
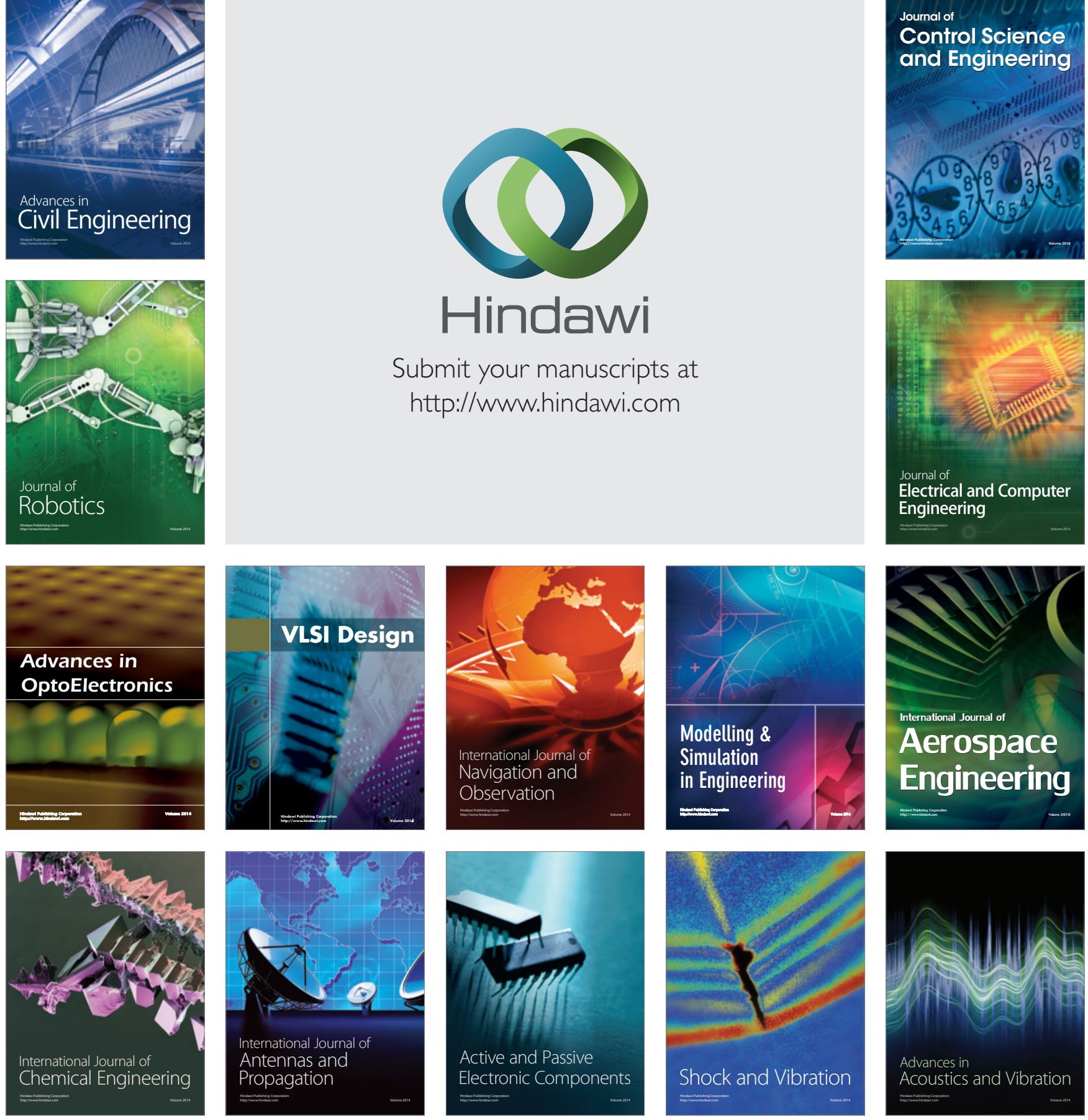